\title{
Not Just Big Kids: Paediatric Intensive Care Nurses' Experience of Working in Adult Intensive Care during the COVID-19 Pandemic in A UK Hospital
}

\author{
Heather Burnett*, Philippa Gibson and Catia Pinto \\ Paediatric Intensive Care, Evelina London Children's Hospital, South Thames Retrieval Service, UK
}

\begin{abstract}
Introduction: The aim of the article was to collect and analyse the experiences of paediatric intensive care nurses redeployed to adult intensive care during COVID-19.

Materials and Methods: A survey was sent to 31 re-deployed paediatric intensive care nurses. The anonymous survey included both open and closed questions allowing space for comments. Framework analysis was carried out to identify themes.

Results: Twenty-five of the nurses who received the survey responded (81\%). The themes motivation, provision of care, teamwork, and wellbeing were identified. Eighteen of the nurses who re-deployed would volunteer to work in adult intensive care again if there were a need.

Discussion: The results of our survey were used alongside existing literature to explore the themes identified above. The effects of re-deployment on paediatric nurses were highlighted and recommendations for future pandemic planning were offered.
\end{abstract}

\section{Introduction}

Our Paediatric Intensive Care Unit (PICU) has over 180 nurses trained in critical care. With the COVID-19 pandemic looming in the UK, PICUs were encouraged to start preparing to take young adults should the Adult Intensive Care Units (AICU) reach capacity [1]. NHS England released guidance [2] on the re-deployment of nurses from adult areas to AICU. There was no mention of paediatric nurses in this guidance, however our PICU re-deployed 40 nurses to AICU on either a full- or part-time basis.In order to prepare our nurses for re-deployment training opportunities were created both online and face-to-face.

The aim of this article is to explore the collective experiences of those PICU nurses re-deployed to AICU during the COVID-19 pandemic. We wanted to identify common themes and reflect on experiences, to enable identification of any need for ongoing support, and to inform future pandemic planning on our PICU.

\section{Materials and Methods}

\section{Design}

A phenomenological approach was taken to facilitate exploration of the lived experiences of the nurses who were re-deployed during the COVID-19 pandemic. This method was chosen as it aims to find meaning in the experiences of individuals [3]. Open questions were used to allow participants to describe their experience.

\section{Ethics}

As the project involved a staff survey only, it was exempt, under National Health Service (NHS) Health Research Authority regulations [4], from the need for IRB (Research Ethics Committee) approval. However, our survey was registered within our department in our hospital.

Participation in the survey was voluntary and anonymous. Participants were informed of the purpose of the survey and

*Corresponding author: Dr. Heather Burnett, Advanced Nurse Practitioner, Paediatric Intensive Care, Evelina, London Children's Hospital, South Thames Retrieval Service, Westminster Bridge Road, London SE1 7EH, UK

Received: July 21, 2020

Accepted: August 26, 2020

Published online: August 28, 2020

Citation: Burnett H, Gibson P, Pinto C (2020) Not Just Big Kids: Paediatric Intensive Care Nurses' Experience of Working in Adult Intensive Care during the COVID-19 Pandemic in A UK Hospital. J Nurs Pract 3(1):143-147 
Citation: Burnett H, Gibson P, Pinto C (2020) Not Just Big Kids: Paediatric Intensive Care Nurses' Experience of Working in Adult Intensive Care during the COVID-19 Pandemic in A UK Hospital. J Nurs Pract 3(1):143-147

that they may be quoted. They were also informed that any information volunteered would not be used for any other purpose. Consent was implied by the completion and return of the survey.

\section{Participants}

Thirty-one of the nurses who volunteered for re-deployment were in an AICU email group; the survey was sent to each member of this group. The 9 nurses not in this email group were excluded. All the nurses who were surveyed fulfilled our hospital's criteria for redeployment and were therefore not further stratified.

\section{Data collection}

A survey was created consisting of 10 questions, both open and closed (with the option for further comments) (Appendix 1). The questions covered topics that the authors felt to be relevant having had multiple informal discussions with the re-deployed nurses. The survey was sent out by email and completed online. The survey was completed over five days in mid-July 2020.

\section{Data analysis}

A framework analysis was carried out [5]. The authors familiarised themselves with the responses and commonalities of experience were identified. These were translated into a Microsoft Excel document and categorised into themes. Pertinent quotations were selected and considered in line with existing pandemic research. All three authors were involved in each stage.

\section{Results}

Twenty-five nurses completed the questionnaire and each nurse answered all questions. Analysis of results generated four themes for discussion.

\section{Motivation}

Staff were motivated to re-deploy as they recognised that they had transferable skills that may be useful in AICU. One stated they wished 'to utilise my PICU skills to assist/support the AICU nurses who had a massively increased workload. I felt that I had a skill that would be a benefit in the pandemic...'. Nurses also felt a duty to re-deploy; one nurse wrote "I wanted to feel like I was contributing at a time of international crisis...'. Additionally, a feeling of guilt was demonstrated: 'I felt it was unfair of adult nurses to be struggling ...', and 'It felt wrong not to'. Nurses did, however, express concerns about re-deploying; twenty nurses (80\%) feared working in a new environment, four were concerned about personal protective equipment and 10 about contracting and spreading the disease.

Another motivation for volunteering was fear that re-deployment would later become mandatory. Seven nurses felt that volunteering allowed them to retain control and alleviate uncertainty. One nurse stated, 'I felt the anxiety of not knowing whether I would move or not move to AICU on a daily basis was stressful and preferred to sign up for a period of time'. A second participant commented, 'I was being sent on an ad hoc basis and felt I would be more comfortable if I knew every day, I was going to AICU rather than finding out on a shift by shift basis'.

\section{Provision of care}

Nineteen respondents (76\%) reported that they felt they were able to provide good patient care. However, in the comments it became apparent that this was not their usual standard of care, but the best they could provide in the circumstances: 'I felt I did the best of my ability as time would allow but would have liked to do more'.

A feeling of frustration emerged due to the lack of time and staff available for the provision of basic nursing care. One nurse stated, '...having 2 ventilated patients all the time was too impossible to do full basic cares to patients especially when there is no help turn or roll the patient. It's frustrating how much I wanted to be handing over the patient in a satisfactory manner, but I can only do what I can at that time...'. Other comments were: "I felt I did the best I could at the time... This was partly due to rushing due to time constraints (you could only do turns when someone was free)...', and 'At the start I did not feel like I did as the units I worked on were so understaffed it felt impossible.'

Communication with families was also seen as important: 'the facetime calls with family were priceless', however lack of time also impacted on this. One nurse stated, 'I really felt that I couldn't give enough time to relatives who were phoning for updates.'

After the first few weeks, nurses from non-critical care backgrounds were re-deployed to provide support to the bedside team. This allowed more time for patient care and improved nurses' feelings about care provision. One nurse wrote that once she had some support, 'we were able to catch up on basic cares like catheter care, skin care etc that may have been missed on busier shifts.' Another nurse acknowledged the difference that having time made to their patient care, 'the little things make all the difference - holding a patient's hand, making time to talk to them (I was lucky I had the time to do this)'.

\section{Teamwork}

There was an overwhelmingly positive response to the collaboration between the paediatric and adult teams. A positive experience reported by one of the PICU nurses was '...making connections and friendships with my fellow adult nurse colleagues'. This teamwork seems to have been in place from the outset: 'I think what we done was amazing in terms of how we pulled off the setting up of the place'. Other words used by the PICU nurses to describe the AICU nurses included 'lovely', 'friendly and helpful', and 'great'. One respondent in this survey even discussed how the collaboration could be of future benefit: '... I think we could bring practice from both together to improve things further not just if we had a second wave but just daily practice'.

\section{Wellbeing}

The survey asked whether working in AICU affected the 
Citation: Burnett H, Gibson P, Pinto C (2020) Not Just Big Kids: Paediatric Intensive Care Nurses' Experience of Working in Adult Intensive Care during the COVID-19 Pandemic in A UK Hospital. J Nurs Pract 3(1):143-147

nurses' personal lives at the time; 13 respondents (52\%) said yes.

Nine nurses described having experienced emotional distress. One nurse stated that working in AICU 'left me...sad and traumatised'; another stated 'felt like my life was consumed by AICU. Didn't know how to process what was happening...'. Feelings of being overwhelmed and of anxiety were prevalent. One nurse commented that on some days they did not want to go to work, and another said they were 'not sleeping well worrying about care given and anxiety/worry of making mistakes'.

Nurses described feeling 'burnout' due to working extra shifts; one stated 'I knew they needed the staff so I volunteered to do extra'. Staff repeatedly mention feeling 'exhausted' and 'tired', one specifically mentioning how tiring it was finishing late every shift, and another stating "I was so exhausted, both physically and mentally'.

The impact that working on AICU had on family and friends was also mentioned. One participant described needing to provide lots of reassurance to their loved ones that they would be safe. The emotional toll working in AICU had on the nurses in turn also impacted on their families: 'The stress and exhaustion left me feeling irritable and sometimes angry when at home'; 'the emotional toll meant my husband saw how upset I was most days...'.

When asked if having worked on AICU continues to affect their personal life, six nurses reported that it does. The adaption to a 'new normal' was discussed, including improvement in the way they look after themselves and in their infection control practices. Ongoing emotional distress was also mentioned: 'Still don't know how to process it', 'have had very vivid and upsetting nightmares...', 'slightly burnt out still'.

Nurses were asked which of the mechanisms of support available they found useful. Twenty-two nurses (88\%) found being in a bay with other PICU nurses supportive. In addition to those who were re-deployed, on occasion extra PICU nurses would go to AICU to help with morning cares and turns. One of the re-deployed nurses commented, 'it was brilliant when PICU nurses came as a turning team. Just seeing a familiar face brightened the shift'. Later when asked about engagement with formal debriefing, one respondent stated that they 'gained a lot of support from fellow PICU volunteers to AICU'. Additionally, 16 nurses found the provision of food (lunch was delivered every day) helpful.

Several group debriefing opportunities led by a psychologist were organised for those who re-deployed (with virtual options) both during and after re-deployment. The survey showed that 10 nurses had engaged with debriefing, and comments showed a mixed response regarding the effectiveness of this.

Several nurses commented on how helpful it was to find out that others were feeling the same as them, one stating 'didn't feel alone hearing that others were struggling too'. Some other responses included: 'I personally don't find formal group debrief session useful', 'debrief not overly helpful... psychologist didn't offer much support'.
A challenge that was seen in the responses was accessing the debriefs: 'Attempted to dial in many times', 'hard not being in the room', 'asked to leave as was only able to join after exact start time'. One nurse stated that she had stayed on PICU to enable others to attend and two nurses stated that they had not been made aware of the debriefing opportunities.

Despite the challenges, when asked 18 nurses (72\%) said that they would volunteer again should there be the need. One nurse summed up her experience: 'Felt proud that I was able to contribute and be part of the unbelievable pandemic time that I have never experience in my entire career'.

\section{Discussion}

From our survey the motivation to re-deploy felt by nursing staff seems to be explained not just by their legal duty as registered nurses, but also by their desire to help colleagues in AICU and to contribute at a time of need. A systematic review of nurses' experiences [6] found that in situations of crisis the sense of duty and commitment to patients as well as to multi-disciplinary relationships can overcome the sense of fear and stress; from our survey this clearly seems to be the case.

Nielsen and Dieperink [7] tried to explain this phenomenon by putting nurses into three different categories: The 'concerned', the 'noise makers', and the 'devoted'. The 'devoted' feel their duty to provide compassionate care goes beyond their own personal safety. They understand how important their skills are. From our survey it cannot be concluded whether most nurses fit into the 'devoted' group, but certainly it seems that they volunteered for re-deployment despite their concerns about working in a new environment, the provision of PPE, and contracting and spreading the disease.

Frustration was felt at not being able to provide the desired level of basic nursing care. No literature was found exploring the impact that being unable to provide this care has on the nurses themselves. The fact that respondents chose to comment on the challenge of providing basic physical care suggests that being unable to did indeed affect them.

Paediatric nurses in particular are used to providing family centred care [8] and disappointment was felt when it was not possible to provide this. The comments made about the importance of the family face time calls reiterate the importance to PICU nurses of having the time and support to deliver not only basic physical care, but also family centred care.

In our hospital the COVID-19 pandemic initiated a novel collaboration between PICU and AICU. This teamwork was perceived by the staff as a positive experience. Kneyber, et al. [9] described similar; their paediatric and adult clinical teams prospered in the COVID-19 pandemic, developing strong professional relationships. The suggestion made by one respondent in our survey that adult and paediatric teams working together could be of benefit in daily practice as well as in a 'second wave' may be worthy of consideration in our hospital's pandemic planning as this may encourage the establishment of strong professional relationships in preparation. 
Guidance and recommendations focusing on the wellbeing of staff emerged [10-13] as the COVID-19 pandemic progressed, acknowledging that without adequate preparation and support some healthcare workers would be at risk of psychological injury for a significant amount of time [12,14], found that the mental health of nurses directly involved in patient care during a pandemic was especially at risk, however Williams, et al. [11] state that adequate support mechanisms have been shown to reduce the risk of exposure to such events.

Both formal and informal peer supports are of great value $[10,11]$ suggest that on each shift staff should pick a 'buddy' - someone they know and trust, who can be confided in and offer support if needed. Although this process was not formalised it seems that collectively the PICU nurses informally provided this support to each other. Cole-King and Dykes [10] also state that debriefings which encourage the discussion of distressing feelings must be avoided as they risk causing psychological harm to staff. Although some nurses explicitly stated that they did not find the formal debriefings helpful, others saw them as an opportunity to engage with peer support, suggesting that they may have been be of some benefit to some nurses. Accessing the debriefs was reported as challenging; this may have encouraged peer support as it is likely this was more readily available.

In addition to peer support, the provision of food daily was valued support mechanism. This is supported by literature from the H1N1 pandemic where the provision of food for staff had a positive impact on team morale [15].

This article has explored the experiences of PICU nurses re-deployed to AICU during the COVID-19 pandemic. The impact of re-deployment on staff was highlighted as were the effective support mechanisms. The value of peer support and team working was a common thread throughout the responses. These enabled nurses to provide better patient care and were a key motivator for many volunteers as they felt the need to support colleagues. Respondents also found peer support useful in the debriefing sessions.

It is vital that we learn from these nurses' experiences. It is evident from our survey that nurses were and are deeply affected by their time on AICU. It is also evident that peer support is essential and as many of the nurses in our survey are still affected we have already instigated further peer support. Recommendations for future pandemic planning are re-deployment which involves a formalised buddy system, as well as continuing educational and managerial support. In addition, information about support systems, including the ongoing provision of food, should be widely communicated and followed up.

\section{Limitations}

This survey was conducted in a tertiary referral hospital and only small cohorts of nurses were surveyed, limiting the statistical significance of the results. Nurses were not stratified according to gender, age, experience and seniority.

\section{Acknowledgements}

The authors wish to acknowledge the bravery and commitment of all PICU nurses who volunteered to work in AICU during the COVID-19 pandemic, and of our AICU colleagues. The authors also wish to acknowledge all the nurses that kept PICU running whilst their colleagues were redeployed. The authors would like to thank all those that completed the survey and shared their experiences so honestly, and finally would like to thank Dr Shane Tibby.

\section{References}

1. (2020) Paediatric intensive care society and the intensive care society. Joint position statement.

2. (2020) NHS. COVID-19: Deploying our people safely.

3. Sinuff T, Cook DJ, Giacomini M (2007) How qualitative research can contribute to research in the intensive care unit. Journal of Critical Care 22: 104-111.

4. (2020) NHS Health Research Authority.

5. Ward DJ, Furber C, Tierney S, et al. (2013) Using framework analysis in nursing research: A worked example. Journal of Advanced Nursing 69: 2423-2431.

6. Fernandez R, Lord H, Halcomb E, et al. (2020) Implications for COVID-19: A systematic review of nurses' experiences of working in acute care hospital settings during a respiratory pandemic. International Journal of Nursing Studies.

7. Nielsen D, Dieperink KB (2020) Cultural perspectives and nurses reactions on the corona pandemic: A critical view from Denmark. Journal of Transcultural Nursing 31: 333-336.

8. Butler A, Willetts G, Copnell B (2017) Nurses' perceptions of working with families in the paediatric intensive care unit. Nursing in Critical Care 22: 195-202.

9. Kneyber MC, Engels B, van der Voort PH (2020) Paediatric and adult critical care medicine: Joining forces against Covid-19. Critical Care 24: 1-2.

10. Cole-King A, Dyke L (2020) Staff wellbeing during COVID-19: A structured approach for healthcare organisations.

11. Williams R, Murray E, Neal A et al. (2020) Top ten messages for supporting healthcare staff during the COVID-19 pandemic.

12. Greenberg N, Docherty M, Gnanapragasam S, et al. (2020) Managing mental health challenges faced by healthcare workers during covid-19 pandemic. bmj 368.

13. Walton M, Murray E, Christian MD (2020) Mental health care for medical staff and affiliated healthcare workers during the COVID-19 pandemic. Eur Heart J Acute Cardiovasc Care 9: 241247.

14. Brooks SK, Dunn R, Amlôt R, et al. (2018) A systematic, thematic review of social and occupational factors associated with psychological outcomes in healthcare employees during an infectious disease outbreak. J Occup Environ Med 60: 248-257.

15. Corley A, Hammond NE, Fraser JF (2010) The experiences of health care workers employed in an Australian intensive care unit during the H1N1 Influenza pandemic of 2009: A phenomenological study. Int J Nurs Stud 60: 577-585. 


\section{Appendix I}

Questions from the survey:

1) Why did you volunteer to work in AICU during the pandemic?

2) What were your main concerns before you went? Tick all that apply.

- The provision of PPE

- Contracting and spreading the illness to your household or colleagues

- The transferability of your PICU skills

- Working in an unknown environment with an unfamiliar team

- Caring for a dying adult and performing last offices

- Being overwhelmed with the workload

- Other (comment box available)

3) Did you access any training for AICU? If so, was it helpful?

If you answered yes to the previous question, please comment further if you wish.

4) Did you feel like you provided good patient care?

If you answered yes to the previous question, please comment further if you wish.

5) Did you find any of the following supportive whilst you were in AICU? Tick all that apply.

- Provision of food

- Wellbeing centre

- Being in a bay with other PICU nurses

- Access to psychologic support

- Support from education/management team

- Other (comment box available)

6) Did working in AICU affect your personal life at the time?

If you answered yes to the previous question, please comment further if you wish.

7) Does having worked in AICU continue to impact on your personal life?

If you answered yes to the previous question, please comment further if you wish.

8) Give three positive things that you have taken away from your time in AICU

9) Did you engage with any of the debriefing opportunities?

Please expand below if you wish.

10) If a second wave comes, would you volunteer to go again?

DOI: $10.36959 / 545 / 375$ 